\title{
A COMPLEX OF PROBLEMS PROPOSED BY POST
}

\author{
BY WILSON E. SINGLETARY ${ }^{1}$
}

Communicated by Leon Henkin, October 4, 1963

There are eight theorems contained in this note. Theorems 1 and 7 are solutions of problems concerning fragments of the propositional calculus which were proposed by Tarski in 1946 at the Princeton Bicentennial. These problems were solved by Post and Linial ${ }^{2}$ in 1948. Theorems 2, 4-6 and 8 are solutions of a complex of problems proposed by Post to Boone in the spring of 1948. Theorem 3 is the solution of a problem which was suggested to the writer as a natural consequence of the proof of Theorem 2. The writer became acquainted with these problems of Post while working under the direction of Boone. The methods used in the detailed proofs of these theorems, which will be published at a later date, were inspired by M. K. Yntema's ${ }^{3}$ detailed and elegant proofs of the Post-Linial Theorems. Yntema's work was in turn suggested by an outline of the proofs given by Davis. ${ }^{4}$ The writer understands from Boone that other detailed proofs of the Post-Linial Theorems will be forthcoming from Ronald Harrop and M. D. Gladstone. The proofs of these theorems are outlined here for completeness, because their machinery is an integral part of the proofs of the other theorems, and also because they differ somewhat from those of Yntema. All proofs of the present theorems are constructive.

A partial propositional calculus is a system having $\sim, \supset,[$, and ] as primitive symbols along with propositional variables $p_{1}, q_{1}, r_{1}, p_{2}$, $q_{2}, r_{2}, \ldots$. Its well-formed formulas are (1) a propositional variable, (2) $[A \supset B]$, where $A$ and $B$ are well-formed formulas, and (3) $\sim A$ where $A$ is a well-formed formula. It has a finite set of tautologies as axioms and its two rules of inference are modus ponens and substitution. If the restriction requiring all of the axioms to be tautologies be dropped, the resulting system will be called a generalized partial propositional calculus.

A semi-Thue system ${ }^{5}$ is specified by a finite alphabet $\mathbb{Z}$ and a finite set of word pairs $\mathfrak{u}$.

1 Research partially supported by N.S.F. Grant No. GP-1568.

See [3].

3 See [4].

4 See [2, pp. 137-142].

5 There are two viewpoints possible concerning semi-Thue systems. The one given here was chosen because the rules more closely resemble the axioms of the generalized partial propositional calculi used. For the two formulations see [1] and [4]. These are easily shown to be equivalent as pointed out in [4]. 


$$
\begin{aligned}
& \text { B: } a_{1}, a_{2}, \cdots, a_{n}, \\
& \mathfrak{U}: A_{1} \rightarrow B_{1}, A_{2} \rightarrow B_{2}, \cdots, A_{m} \rightarrow B_{m} .
\end{aligned}
$$

A word is a finite string of symbols of $\mathbb{Z}$, with possible repetitions, which may be empty. $C \vdash D$, where $C$ and $D$ are words on $B$ shall mean there exists a finite sequence of statements $C_{1} \vdash D_{1}, C_{2} \vdash D_{2}$, $\cdots, C_{l} \vdash D_{l}$ such that $C_{l}$ is $C$ and $D_{l}$ is $D$, and such that each statement $C_{i} \vdash D_{i}$ is justified by one of the following rules:

1. If $C \vdash D$, then $A C \vdash A D$;

2. If $C \vdash D$, then $C A \vdash D A$;

3. $C \vdash C$;

4. If $C \rightarrow D$, then $C \vdash D$;

5. If $C \vdash E$ and $E \vdash D$, then $C \vdash D$.

It is well known ${ }^{6}$ that there exists a semi-Thue system on two letters which has an unsolvable word problem and no defining relation of which is of the form $A \rightarrow B$ where either $A$ or $B$ is empty.

THEOREM 1. There exists a partial propositional calculus with a recursively unsolvable decision problem.

Let $\sigma$ be a semi-Thue system with an unsolvable word problem defined by:

$$
\begin{aligned}
& 3_{\sigma}: 1, b, \\
& \mathfrak{U}_{\sigma}: G_{i} \rightarrow \bar{G}_{i}, i=1,2, \cdots, m
\end{aligned}
$$

where no $G_{i}$ or $\bar{G}_{i}$ is the empty word.

If $W$ is a word of $\sigma$, then define $W^{\prime}$ to be the well-formed formula of the propositional calculus given by

$$
\begin{aligned}
& 1^{\prime} \text { is } \sim \sim\left[\sim p_{2} \supset \sim p_{2}\right] \\
& b^{\prime} \text { is } \sim \sim \sim \sim\left[\sim p_{2} \supset \sim p_{2}\right] \\
& (V 1)^{\prime} \text { is }\left[V^{\prime} \& 1^{\prime}\right], \text { and } \\
& (V b)^{\prime} \text { is }\left[V^{\prime} \& b^{\prime}\right],
\end{aligned}
$$

where $A \& B$ is an abbreviation for $\sim[A \supset \sim B]$.

Now specify a partial propositional calculus ${ }^{7} \mathfrak{B}_{\odot}$ by the following set of axioms.

1. $\left[p_{1} \&\left[q_{1} \& r_{1}\right]\right] \supset\left[\left[p_{1} \& q_{1}\right] \& r_{1}\right]$,

2. $\left[\left[p_{1} \& q_{1}\right] \& r_{1}\right] \supset\left[p_{1} \&\left[q_{1} \& r_{1}\right]\right]$,

3. $\left[p_{1} \supset q_{1}\right] \supset \square\left[r_{1} \& p_{1}\right] \supset\left[r_{1} \& q_{1}\right]$,

${ }^{6}$ See $[2$, p. 93].

${ }^{7}$ This is the same system used by Yntema in [4] with the addition of axiom 8 . Cf. [2]. 
4. $\left[p_{1} \supset q_{1}\right] \supset=\left[p_{1} \& r_{1}\right] \supset\left[q_{1} \& r_{1}\right]$,

5. $p_{1} \supset p_{1}$,

6. $G_{i}^{\prime} \supset \bar{G}_{i}^{\prime}, i=1,2, \cdots, m$,

7. $\left[p_{1} \supset q_{1}\right] \supset\left[q_{1} \supset r_{1}\right] \supset\left[p_{1} \supset r_{1}\right]$,

8. $\sim\left[p_{1} \supset p_{1}\right] \supset q_{1}$.

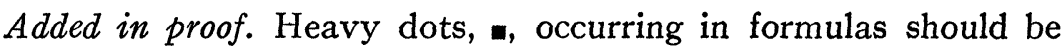
interpreted to have the meaning assigned to them in Church's Introduction to mathematical logic, p. 15. But the reader can, for all expressions used in the present note, get the effect of this convention simply by replacing each heavy dot by a left bracket and adding a corresponding right bracket at the end of the expression. We have also adopted the convention of omitting outer brackets.

If $X$ is a well-formed formula of $\mathfrak{P}_{\sigma}$, then $X$ is regular if (1) $X$ is $1^{\prime}$, or $X$ is $b^{\prime}$, or (2) $X$ is of the form $\left[X_{1} \& X_{2}\right]$, where $X_{1}$ and $X_{2}$ are regular well-formed formulas.

If $X$ is regular, then $\langle X\rangle$ is the unique word of $\sigma$ obtained by the following procedure: (1) abbreviating $X$ so that it contains only [ , ], $\&, 1^{\prime}$, and $b^{\prime},(2)$ removing all occurrences of $[$,$] , and \&,(3)$ replacing $1^{\prime}$ by 1 , and $b^{\prime}$ by $b .{ }^{8}$

If $W$ is a well-formed formula of $\mathfrak{B}_{\sigma}$, then $W$ is valid ${ }^{9}$ if and only if $W$ is of the form $W_{1} \supset W_{2}$ and (1) $W_{1}$ is regular, $W_{2}$ is regular, and $\left\langle W_{1}\right\rangle \vdash_{\sigma}\left\langle W_{2}\right\rangle$, or (2) $W_{1}$ is not regular, and if $W_{1}$ is valid then $W_{2}$ is valid.

It can be proved that if $X \vdash{ }_{\sigma} W$, then $\vdash_{\Re_{\sigma}} X^{\prime} \supset W^{\prime}$ and also that all theorems of $\mathfrak{F}_{\sigma}$ are valid and hence if $X$ and $W$ are regular and $\vdash \Re_{\sigma} X \supset W$, then $\langle X\rangle \vdash_{\sigma}\langle W\rangle$. From this we have $W_{1} \vdash_{\sigma} W_{2}$ if and only if $\vdash_{\beta_{\sigma}} W_{1}^{\prime} \supset W_{2}^{\prime}$ and consequently $\Re_{\sigma}$ has a recursively unsolvable decision problem.

Definition (Post). A well-formed formula $W$ of a partial propositional calculus $\mathfrak{B}$ is completely untrue with respect to $\mathfrak{P}$ if there is no substitution instance $V$ of $W$ such that $V$ is a theorem of $\Re$.

THEOREM 2. For the partial propositional calculus $\mathfrak{B}_{\sigma}$ the problem to determine of an arbitrary wff $W$ of $\mathfrak{B}_{\sigma}$ whether or not $W$ is completely untrue with respect to $\mathfrak{P}_{\sigma}$ is recursively unsolvable.

We shall write $X=W^{A}$ to mean that there is a regular wff $W$ and a wff $A$ such that $X$ is the result of substituting $A$ for $p_{2}$ in $W$. We shall say that $X$ is $A$-regular if $X=W^{A}$ for some $W$.

${ }^{8}$ Cf. $[2 ; 4]$.

- This definition differs slightly from Yntema's [4] and allows some simplification of the verification of the validity of the theorems. 
If $X$ is a wff of $\mathfrak{B}_{\sigma} X$ is $A$-valid if $X$ is of the form $X_{1} \supset X_{2}$ and (1) $X_{1}$ $=V_{1}^{A}, X_{2}=V_{2}^{A}$ and $\vdash_{\mathfrak{B}_{\sigma}} V_{1} \supset V_{2}$, or (2) $X_{1}$ is not $A$-regular, and if $X_{1}$ is $A$-valid then $X_{2}$ is $A$-valid. ${ }^{10}$

It can be proved that all theorems of $\mathfrak{B}_{\sigma}$ are $A$-valid and hence that $\vdash_{\Re_{\sigma}} V_{1}^{A} \supset V_{2}^{A}$ if and only if $\vdash_{\beta_{\sigma}} V_{1} \supset V_{2}$.

THEOREM 3. The problem to determine whether or not two partial propositional calculi have a theorem in common is recursively unsolvable.

Let $\Re_{\sigma}$ be the partial propositional calculus of Theorem 1. Let $\mathfrak{P}\left(W_{1}, W_{2}\right)$ * be the partial propositional calculus having the single axiom $W_{1} \supset W_{2}$, where $W_{1}$ and $W_{2}$ are regular wffs of $\mathfrak{P}_{\sigma}$. Then $\mathfrak{P}_{\sigma}$ and $\mathfrak{P}\left(W_{1}, W_{2}\right)$ * have a theorem in common if and only if $\vdash_{\Re_{\sigma}} W_{1} \supset W_{2}$.

Definition. A partial propositional calculus $\mathfrak{B}$ is inconsistent in the sense of Post if a wff consisting of a propositional variable alone is a theorem of $\mathfrak{P}$.

Definition (Post). Wff $W$ of a partial propositional calculus $\mathfrak{P}$ is false with respect to $\mathfrak{P}$ if $\mathfrak{P}$ becomes inconsistent in the sense of Post upon the addition of $W$ to the axioms of $\mathfrak{B}$.

TheOREM 4. For the partial propositional calculus $\mathfrak{B}_{\sigma}$ the problem to

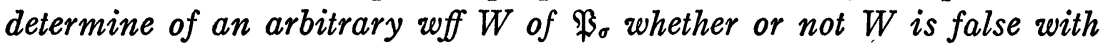
respect to $\mathfrak{B}_{\sigma}$ is recursively unsolvable.

It can be proved for the system $\mathfrak{B}_{\sigma}$ of Theorem 1 that $\left[W_{1} \supset W_{2}\right]$ $\supset\left[\sim\left[p_{1} \supset p_{1}\right]\right]$, where $W_{1}$ and $W_{2}$ are arbitrary regular wffs of $\Re_{\sigma}$, is false with respect to $\mathfrak{P}_{\sigma}$ if and only if $\vdash_{\Re_{\sigma}} W_{1} \supset W_{2}$.

TheOREM 5. The problem to determine of a generalized partial propositional calculus $\mathfrak{P}$ whether or not $\mathfrak{P}$ is inconsistent in the sense of Post is recursively unsolvable.

Let $W_{1}$ and $W_{2}$ be arbitrary regular wffs of the system $\mathfrak{B}_{\sigma}$ of Theorem 1 , and let $\mathfrak{P}\left(W_{1}, W_{2}\right)$ be $\mathfrak{P}_{\sigma}$ with the added axiom $\left[W_{1} \supset W_{2}\right]$ $\supset\left[\sim\left[p_{1} \supset p_{1}\right]\right]$. Then it is easily proved that $\mathfrak{B}\left(W_{1}, W_{2}\right)$ is inconsistent in the sense of Post if and only if $\vdash_{\Re \sigma} W_{1} \supset W_{2}$.

Definition (Post). Wff $W$ is completely false with respect to a partial propositional calculus $\mathfrak{B}$ if for every substitution instance, $V$, of $W, V$ is false with respect to $\mathfrak{B}$.

THEOREM 6. For the partial propositional calculus $\mathfrak{B}_{\sigma}$ the problem to determine of an arbitrary wff $W$ of $\mathfrak{B}_{\sigma}$ whether or not $W$ is completely false with respect to $\mathfrak{B}_{\sigma}$ is recursively unsolvable.

${ }^{10}$ This definition differs slightly from Yntema's [4] and allows some simplification of the verification of the $A$-validity of the theorems. 
It can be proved for the system $\mathfrak{B}_{\sigma}$ of Theorem 1 that if $W_{1}$ and $W_{2}$ are arbitrary regular wffs of $\mathfrak{P}_{\sigma}$, then $\left[W_{1} \supset W_{2}\right] \supset\left[\sim\left[p_{1} \supset p_{1}\right]\right]$ is completely false with respect to $\mathfrak{B}_{\sigma}$ if and only if $\vdash_{\mathfrak{B}_{\sigma}} W_{1} \supset W_{2}$.

Definition (Post). Wff $W$ of a partial propositional calculus $\mathfrak{B}$ is untrue with respect to $\mathfrak{P}$ if $W$ is not a theorem of $\mathfrak{B}$.

Definition (Post). A partial propositional calculus $\mathfrak{B}$ is closed if upon the addition of any untrue wff to the axioms of $\mathfrak{P}, \mathfrak{P}$ becomes inconsistent in the sense of Post.

THEOREM 7. The problem to determine of an arbitrary partial propositional calculus $\mathfrak{B}$ whether or not $\mathfrak{B}$ is closed is recursively unsolvable.

Let $W_{1}$ and $W_{2}$ be arbitrary regular wffs of the system $\mathfrak{P}_{\sigma}$ of Theorem 1 . Let $\mathfrak{B}\left(W_{1}, W_{2}\right)^{\prime}$ be the system obtained by adding the following wffs to the axioms of $\mathfrak{B}_{\sigma}$.

$$
\begin{aligned}
& {\left[W_{1} \supset W_{2}\right] \supset p_{1} \supset\left[q_{1} \supset p_{1}\right],} \\
& {\left[W_{1} \supset W_{2}\right] \supset \cdot\left[p_{1} \supset\left[q_{1} \supset r_{1}\right]\right] \supset\left[p_{1} \supset q_{1}\right] \supset\left[p_{1} \supset r_{1}\right],} \\
& {\left[W_{1} \supset W_{2}\right] \supset \cdot\left[\sim q_{1} \supset \sim p_{1}\right] \supset\left[p_{1} \supset q_{1}\right] .}
\end{aligned}
$$

Then it can be proved that $\mathfrak{P}\left(W_{1}, W_{2}\right)^{\prime}$ is closed if and only if $\vdash_{\Re_{\sigma}} W_{1} \supset W_{2}$.

Definition (Post). A partial propositional calculus $\mathfrak{B}$ is completely closed if $\mathfrak{B}$ becomes inconsistent in the sense of Post upon the addition of any completely untrue wff to the axioms of $\mathfrak{B}$.

THEOREM 8. The problem to determine of an arbitrary partial propositional calculus $\mathfrak{B}$ whether or not $\mathfrak{B}$ is completely closed is recursively unsolvable.

It can be shown that a system $\mathfrak{B}\left(W_{1}, W_{2}\right)^{\prime}$ of Theorem 7 is completely closed if and only if it is closed.

\section{REFERENCES}

1. William W. Boone, The word problem, Ann. of Math. (2) 70 (1959), 207.

2. Martin Davis, Computability and unsolvability, McGraw-Hill, New York, 1958.

3. Samuel Linial and Emil L. Post, Recursive unsolvability of the deducibility, Tarski's completeness and independence of axioms problems of the propositional calculus, Bull. Amer. Math. Soc. 55 (1949), 50. (Abstract)

4. M. K. Yntema, $A$ detailed argument for the Post Linial theorems, Notre Dame J. Formal Logic 5 (1964), no. 1.

UNIVERSITY OF ILLINOIS 